\title{
ERRATUM, VOLUME 75
}

A. Ben-Israel, A. Charnes and K. O. Kortanek, Duality and asymptotic solvability over cones, pp. 318-324.

There are eleven possible duality states and not just ten, as stated incorrectly in the theorem. The eleventh state is when both dual problems are INC, PAC and AUBD. An example for this state is our example, p. 323 bottom, but with $\alpha=+1$. and $c=\left(\begin{array}{llll}0 & 1 & 0 & 0\end{array}\right)$. Accordingly, remark (iv) p. 324 is incorrect. This example was communicated to us by C. Kallina and A. C. Williams. An earlier example by Gustafson-Kortanek-Rom appeared in Non-Čebyševian moment problems, Technical Report No. 70, Cornell University, April 1969, p. 8.

We are grateful to Kallina and Williams for this correction. 